\title{
Anticentral and Antisemicentral Elements of Infinite Soluble Groups
}

\author{
Bertram Arthur Frederick Wehrfritz ${ }^{1}$
}

Received: 28 March 2017 / Revised: 15 August 2017 / Accepted: 17 September 2017 /

Published online: 2 December 2017

(C) The Author(s) 2017. This article is an open access publication

\begin{abstract}
An element $a$ of a group $G$ is said to be anticentral in $G$ if $G^{\prime}=\{[g, a]: g \in G\}$ and antisemicentral in $G$ if $\{[g, a]: g \in G\}$ contains a normal subgroup of $G$ of finite index in $G^{\prime}$. We study such elements in various types of infinite soluble group.
\end{abstract}

Keywords Infinite soluble group · Nilpotent group · Locally nilpotent group · Anticentral elements

Mathematics Subject Classification (2010) $20 \mathrm{~F} 16 \cdot 20 \mathrm{~F} 18 \cdot 20 \mathrm{~F} 19$

\section{Introduction}

The notion of an anticentral element of a group has been around for a decade, though I have been unaware of it until very recently. The earliest published work on it, as far as I know, is Ladisch [4]. Most of the past work concerns finite groups, but it turns out that some work of mine in a different context is relevant for extending this to certain types of infinite groups.

An element $a$ of a group $G$ is anticentral if $a G^{\prime}=a^{G}$, equivalently if $G^{\prime}=\{[g, a]$ : $g \in G\}$. This concept lies somehow between the notions of a fixed-point-free (fpf for short) automorphism and a splitting automorphism. Suppose $G=\langle a>H$ is the split extension of its normal subgroup $H$ by the cyclic group $\langle a>$. Let $\alpha \in$ Aut $G$ denote conjugacy on $G$ by $a$. Define maps $\gamma=\gamma(\alpha)$ and $\psi_{i}=\psi_{i}(\alpha)$ of $G$ into itself for integers $i \geq 1$ by

$$
g \gamma=[g, a]=g^{-1} \cdot g \alpha \text { and } g \psi_{i}=g \cdot g \alpha \cdots g \alpha^{i-1} .
$$

Generally, when working with a group $G$ and its automorphism group we actually work in the holomorph of $G$. Thus, for example, in the above $g^{-1} \cdot g \alpha$ actually is the commutator

Bertram Arthur Frederick Wehrfritz

b.a.f.wehrfritz@qmul.ac.uk

1 School of Mathematical Sciences, Queen Mary University of London, London E1 4NS, UK 
$[g, a]$ in the usual sense but in the holomorph of $G$. Note that $\gamma$ need not be a homomorphism. In spite of this, we still use the symbol ker $\gamma$ for $\{g \in G: g \gamma=1\}$. A similar remark applies to $\psi_{i}$.

If $H \psi_{n}=\{1\}$, we say $\alpha$ is a $n$-splitting of $H$ and if $\alpha$ has order $n$ with $H \psi_{n}=\{1\}$ we just say $\alpha$ is a splitting of $H$. Suppose $\alpha$ has order $n$ on $H$ and order $m$ on $G^{\prime}$, so $m$ divides $n$. All the following are presumably well known.

Lemma 1 Suppose $H$ is finite and consider the following four statements.

a) $\quad \alpha$ acts fpf ${ }^{d y}$ on $G^{\prime}$.

b) $a$ is an anticentral element of $G$; equivalently $G^{\prime}=G \gamma$.

c) $\alpha$ yields an $n$-splitting automorphism of $G^{\prime}$; equivalently $G^{\prime} \psi_{n}=\{1\}$.

d) $\alpha$ yields a splitting automorphism of $G^{\prime}$; equivalently $G^{\prime} \psi_{m}=\{1\}$.

Then a) implies b) and d), b) implies c) and d) implies c). There are no further implications in general between a), b), c) and d).

Here we consider how far these implications extend to infinite groups. (For locally finite groups, these and related questions have already been considered in [1] and [2]). The connections between b), c), and d) are easy to resolve, are quite general, and hence are uninteresting (but see Lemmas 2 and 3 below). However, the implications of a) are much more delicate, restricted and interesting. One cannot be too ambitious because of the following easy example. Let $H$ be the free group of rank $n \geq 2$ on $x_{1}, x_{2}, \ldots, x_{n}$ and $\alpha$ the automorphism of $H$ that permutes the $x_{i}$ cyclically. Then $\alpha$ acts fpf ${ }^{l y}$ on $H$ but is not anticentral in $G=<\alpha>H$; for $y=\left[x_{2}^{2}, x_{1}\right] \in G^{\prime} \backslash G \gamma$; also $y \psi_{n} \neq 1$. In fact $y \psi_{r} \neq 1$ for all $r \geq 1$. (We only need the square if $n=2$ ).

If we weaken our assumptions in a) to $\alpha$ being almost fpf (afpf for short), meaning that $C_{H}(\alpha)$ is finite, in b) to $\alpha$ (or equivalently the element $a$ ) being antisemicentral, meaning that $G \gamma$ contains a normal subgroup of $G$ of finite index in $G^{\prime}$ and c) to $\alpha$ inducing an $n$-splitting on a normal subgroup of $G$ of finite index in $G^{\prime}$, then our conclusions become much more general. They are also more symmetric than the finite case above.

Theorem 1 Let $H$ be a group, $\alpha$ an automorphism of $H$ and $G$ the split extension of $H$ by $<\alpha>$.

a) If $H$ is locally nilpotent and if $\alpha$ is fpf and of order 2, then $\alpha$ is an anticentral element of $G$.

b) For each $m>2$, there exists a finitely generated torsion-free nilpotent group $H$ of class

2 and a fpf automorphism $\alpha$ of $H$ of order $m$ such that $\alpha$ is not anticentral in $G$.

c) There exists an abelian-by-(finite cyclic), polycyclic group $H$ and a fpf automorphism $\alpha$ of $H$ of order 2 such that $\alpha$ is not anticentral in $G$.

d) If $H$ is the wreath product of two infinite cyclic groups (so $H$ is torsion-free, finitely generated and metabelian), then $H$ has a fpf automorphism $\alpha$ of order 2 such that $\alpha$ is not anticentral in $G$.

e) If $H$ is abelian, then $\alpha$ is anticentral in $G$.

Part e) is very easy; we have only included it to point out that we cannot reduce nilpotent of class 2 in b) or metabelian in c) or d) just to abelian. In passing, we remark that in the notation above and of Theorem 1 in particular, the condition $H=H \gamma$ is much stronger than the condition $G^{\prime}=G \gamma$, even although always $G^{\prime} \leq H$ and $G \gamma=H \gamma$. For example, 
if $H$ is finitely generated and soluble-by-finite (actually finitely generated hyper (abelian or finite) would do), if $\alpha$ has finite prime order $p$ and if $H=H \gamma$, then $H$ is a finite nilpotent $p^{\prime}$-group, see $[6,10.53]$.

An FAR group is a soluble-by-finite group with finite Hirsch number that also satisfies the minimal condition on $p$-subgroups for every prime $p$. See [5], specially Chapter 5, for alternative definitions and basic properties of FAR groups. Note that all soluble-by-finite groups of finite rank and hence all minimax groups and all polycyclic-by-finite groups are FAR groups. The following two theorems are little more than reinterpretations of results in [11] and [10].

Theorem 2 Let $G$ be a nilpotent FAR group, $n$ a positive integer and $a$ an element of $G$ of order dividing $n$. Let $\alpha$ denote conjugation by $a$. The following are equivalent.

a) $\alpha$ induces a afpf automorphism on $G^{\prime}$.

b) $a$ is an antisemicentral element of $G$.

c) There exists a normal subgroup $N$ of $G$ of finite index in $G^{\prime}$ such that $\alpha$ induces an $n$-splitting on $N$.

Theorem 3 Let $G$ be an FAR group, $p$ a prime and a an element of $G$ of order $p$. Let $\alpha$ denote conjugation by $a$. The following are equivalent.

a) $\alpha$ induces a afpf automorphism on $G^{\prime}$.

b) $a$ is an antisemicentral element of $G$.

c) There exists a normal subgroup $N$ of $G$ of finite index in $G^{\prime}$ such that $\alpha$ induces a p-splitting on $N$.

We say that a subset of a group $G$ is of finite index in $G$ if it contains a subgroup of $G$ of finite index. With $G$ and $\alpha$ as in Theorem 2 and using the notation above, the corollary to the theorem of [11] says in particular that the following statements are equivalent: $\operatorname{ker} \gamma$ is finite; $G \gamma$ is of finite index in $G$; $\operatorname{ker} \psi_{n}$ is of finite index in $G ; G \psi_{n}$ is finite. The first three of these applied to the action of $\alpha$ on $G^{\prime}$ and also the very simple Lemma 2 below yield Theorem 2. (Note that any FAR group of finite exponent is finite, so if we have $X \leq Y \leq Z$, where $Z$ is an FAR group, $Y$ is normal in $Z$ and $X$ has finite index in $Y$, then $X$ contains a normal subgroup of $Z$ of finite index in $Y$ ). In a similar way, Theorem 3 follows from [10, Theorem 1].

\section{The Proofs}

Let $G$ be a group, $a$ an element of $G$ of finite order $n$ and denote conjugation by $a$ on $G$ by $\alpha$. With $\gamma$ and $\psi_{r}$ defined as above, we have $G \gamma \psi_{n}=\{1\}$; that is, $G \gamma \subseteq \operatorname{ker} \psi_{n}$. Clearly $G \gamma \subseteq G^{\prime}$. Suppose $\alpha$ restricted to $G^{\prime}$ has order $m$. If $g \in G$, then

$$
g \gamma \psi_{m}=g^{-1} a^{-m} g a^{m}=a^{-m g} a^{m}=\left[g, a^{m}\right] .
$$

Now $a^{m} \in C_{G}\left(G^{\prime}\right)$, so $g \gamma \psi_{m} \in C_{G}\left(G^{\prime}\right)$. Therefore, $G \gamma \psi_{m} \subseteq \zeta_{1}\left(G^{\prime}\right)$, the centre of $G^{\prime}$. Also $m$ divides $n$, say $m d=n$. Then for $x \in G^{\prime}$ we have $x \psi_{n}=\left(x \psi_{m}\right)^{d}$. We have proved the following.

Lemma 2 With the notation above $G \gamma \psi_{n}=\{1\}, G \gamma \psi_{m} \subseteq \zeta_{1}\left(G^{\prime}\right)$ and $\left(G \gamma \psi_{m}\right)^{d}=\{1\}$. 
Continuing the notation above, if $a$ is anticentral in $G$, then $G^{\prime}=G \gamma$ and if $a$ is only antisemicentral then, there is a normal subgroup $N$ of $G$ of finite index in $G^{\prime}$ with $N \subseteq G \gamma$. The above yields the following.

Lemma 3 If $a$ is anticentral in $G$, then $G^{\prime} \psi_{n}=\{1\}$ and $\alpha$ is an $n$-splitting of $G^{\prime}$. If $a$ is antisemicentral in $G$, then with $N$ as above $\alpha$ is an $n$-splitting of $N$ and

$$
N \psi_{m} \subseteq \zeta_{1}\left(G^{\prime}\right)_{d}=\left\{z \in \zeta_{1}\left(G^{\prime}\right): z^{d}=1\right\} .
$$

Lemma 4 With the notation above, if $G$ is finite and if a), b), c) and d) are as below, then a) implies b) and d), b) implies c) and d) implies c).

a) $\alpha$ is fpf on $G^{\prime}$.

b) a is anticentral in $G$.

c) $G^{\prime} \psi_{n}=\{1\}$.

d) $G^{\prime} \psi_{m}=\{1\}$.

Proof If $\alpha$ is fpf on $G^{\prime}$, then from [3, 10.1.1] we have $G^{\prime}=G^{\prime} \gamma \subseteq G \gamma \subseteq G^{\prime}$, so $G^{\prime}=G \gamma$ and hence a) implies b). Lemma 3 yields that b) implies c). Since $x \psi_{n}=\left(x \psi_{m}\right)^{d}$ for all $x$ in $G^{\prime}$, so d) implies c). Finally, a) implies that $<a>\cap G^{\prime}=<1>$ and, via [3, 10.1.1], that $G^{\prime}=G^{\prime} \gamma$. Then Lemma 2 applied to $<a>G^{\prime} /<a^{m}>$ yields that $G^{\prime} \psi_{m}=\{1\}$. Thus a) implies d).

Proof of Lemma 1 By Lemma 4, we have that a) implies b) and d), b) implies c), and d) implies c). We have now only to construct suitable counter examples.

Let $H=<x>$ have order 8 and let $\alpha$ be inversion on $H$. Let $G$ be the split extension of $H$ by $\left\langle\alpha>\right.$ and $a=\alpha$. Then $G^{\prime}=G \gamma=H \gamma=<x^{2}>, m=n=2, x^{4} \alpha=x^{4} \neq 1$ and $H \psi_{2}=\{1\}$. Thus, $a=\alpha$ satisfies b), c) and d) but not a).

Let $H=<x>\times<y, z>$, where $|x|=8,|y|=4,|z|=2$ and $y^{z}=y^{-1}$. Let $\alpha \in$ Aut $H$ invert $\left\langle x>\right.$ and centralize $<y, z>$ and set $G=<\alpha>H$. Then $G^{\prime}=<$ $x^{2}, y^{2}>, m=n=2$ and $G^{\prime} \psi_{2}=\{1\}$. Thus, $a=\alpha$ satisfies c) and d). But $G \gamma=H \gamma=<$ $x^{2}>\neq G^{\prime}$, so $a$ does not satisfy b).

Let $H=<x>\times<y>$, where $x$ and $y$ have order 8. Define the map $\alpha$ of $H$ by $\left(x^{i} y^{j}\right) \alpha=x^{i} y^{i+3 j}$. Then $x \alpha^{2}=x y^{4}, x \alpha^{4}=x$ and $y \alpha^{2}=y$. Thus, $\alpha$ is an automorphism of $H$ of order 4. Then $n=4, G$ has order 256, $G^{\prime}=<y>=G \gamma$ and $\alpha$ satisfies b) and hence c). However $G^{\prime}=<y>$ is centralized by $\alpha^{2}$, so $m=2$ and $y \psi_{2}=y \cdot y \alpha=y^{4} \neq 1$, so $\alpha$ does not satisfy d). That is neither b) nor c) implies d).

Lemma 5 Let $H$ be a locally nilpotent group with a fpf automorphism $\alpha$ of order 2. Then $H$ is abelian and inverted by $\alpha, H \psi_{2}=\{1\}$ and $\alpha$ is a splitting automorphism of $H$.

Proof If $X$ is a finitely generated subgroup of $H$, then so is $Y=<X, X \alpha>$ and, assuming $X \neq<1>, \alpha$ induces an fpf automorphism on $Y$ of order 2 . Thus, we may assume that $H$ is finitely generated (and nilpotent). If $T$ denotes the torsion subgroup of $H$, then $T$ is finite and $H / T$ is torsion-free.

By [8, Theorem 1], the group $H$ has an abelian $\alpha$-invariant normal subgroup $A$ inverted by $\alpha$ and of finite index in $H$. Suppose $T=<1>$. Then $H$ is isomorphic to a unipotent (and hence connected by [7,6.6]) matrix group over the integers, see [7, Point 3, p. 23]. Then the Zariski closure of $A$ in $H$ is $H$ itself and hence $H$ is abelian ([7, 5.11]). In particular $\alpha$ inverts $H$. 
We now induct on the order of $T$. Assume $T \neq<1>$ and set $Z=C_{T}(H)$. Then $Z$ is finite of odd order (since $\alpha$ is fpf), non-trivial and normalized by $\alpha$. Also $\alpha$ inverts $Z$. Further, $\alpha$ induces an fpf automorphism on $H / Z$ by [9, Lemma $13 \mathrm{c}$ )]. By induction $H / Z$ is abelian and inverted by $\alpha$. Let $h \in H$. Then $h \alpha=h^{-1} z$ for some $z \in Z$. Now $z \alpha=z^{-1}$ and $\alpha^{2}=1$. Hence

$$
h=(h \alpha) \alpha=\left(h^{-1} z\right) \alpha=(h \alpha)^{-1} z^{-1}=z^{-1} h z^{-1}=h z^{-2},
$$

since $z$ is central. Therefore $z^{2}=1$. But $Z$ has odd order. Consequently, $z=1, h \alpha=h^{-1}$ and $\alpha$ inverts $H$. The remaining parts of the lemma now follow.

Proof of Theorem 1 Part a). Now $H$ is abelian and inverted by $\alpha$ by Lemma 5 and $G=<$ $\alpha>H$. With $g \gamma=[g, \alpha]$ as usual, for the integer $s$ and $h \in H$ we have $\left(\alpha^{s} \cdot h\right) \gamma=$ $h \gamma=h^{-2}$ and so $G \gamma=H \gamma=H^{2}$. Also $\alpha$ centralizes $H / H^{2}$, so $G / H^{2}$ is abelian and $G^{\prime} \leq H^{2}=G \gamma \subseteq G^{\prime}$. Thus $G^{\prime}=G \gamma$ and $\alpha$ is an anticentral element of $G$.

Part b). Suppose for some $m>2$, we have constructed a suitable $H$ and $\alpha$. Let $d>1$, let $c$ be a primitive $m d$ th root of unity in the complex numbers $\mathbf{C}$ and $\operatorname{set} C=\mathbf{Z}[c] \leq \mathbf{C}$. Then $c$ is a fpf automorphism of $C$ of order $m d$ and additively $C$ is free abelian of finite rank.

Set $K=H \times C$, extend $\alpha$ and $c$ to $K$ by letting $\alpha$ centralize $C$ and letting $c$ centralize $H$ and set $\beta=\alpha c$. Then $\beta$ is a fpf automorphism of $K$ of order $m d$. Since $\alpha$ is not anticentral in $G=<\alpha>H$, there exists $x$ in $G^{\prime}$ such that $x \neq[h, \alpha]$ for any $h$ in $H$. Set $L=<\beta>K$. Then $G^{\prime}=H^{\prime}[H, \alpha]=H^{\prime}[H, \beta] \leq L^{\prime}$. Suppose $x=[y, \beta]$ for some $y$ in $L$. Then $x=[k, \beta]$ for some $k$ in $K$. But $x \in H$, so $x=[h, \alpha]$ for some $h \in H$, a contradiction. Thus $L^{\prime} \neq\{[y, \beta]: y \in L\}$ and hence we have extended our counter example for $m$ to one for $m d, d>1$. Consequently, we now have only to construct examples for every odd prime $p$ and for $2^{2}$. We do these together by giving an example for each $m=p^{r} \geq 3$ and every prime $p$.

Thus let $m=p^{r} \geq 3$ and set $f=\phi(m)=m(p-1) / p$, so $f \geq 2$. Let $\omega$ be a primitive $m$ th root of unity in $\mathbf{C}$ and set $J=\mathbf{Z}[\omega]=\oplus_{0 \leq i<f} \mathbf{Z} \omega^{i} \leq \mathbf{C}$. Now

$$
1+\omega^{m / p}+\omega^{2 m / p}+\cdots+\omega^{(p-2) m / p}+\omega^{f}=0 .
$$

Suppose $1+v=v \omega$, where $v=\sum_{0 \leq i<f} v_{i} \omega^{i}$ and the $v_{i}$ are integers. Then

$$
1+\sum_{0 \leq i<f} v_{i} \omega^{i}=\left(\sum_{0 \leq i<f-1} v_{i} \omega^{i+1}\right)-v_{f-1}\left(\sum_{0 \leq j<p-1} \omega^{j m / p}\right) .
$$

Summing the coefficients and canceling, we obtain $1+v_{f-1}=-(p-1) v_{f-1}$, so $p v_{f-1}=$ -1 . But $v_{f-1}$ is an integer. Therefore, no such $v$ exists.

Let $H$ be the 3 by 3 lower unitriangular group $\operatorname{Tr}_{1}(3, J)$ over $J$. If $h=\left(h_{i j}\right) \in H$, represent $h$ by $h=\left(h_{12}, h_{23}, h_{13}\right)$ since these three entries of $h$ determine $h$. Let $\alpha=$ $\operatorname{diag}\left(\omega^{2}, \omega, 1\right) \in G L(3, J)$. If $y=(u, v, w) \in H$, then $y^{\alpha}=\left(u \omega, v \omega, w \omega^{2}\right)$. In particular, conjugation on $H$ by $\alpha$ is a fpf automorphism of $H$ of order $m$ and $G=<\alpha, H>\leq$ $G L(3, J)$ is the split extension of $H$ by $\langle\alpha\rangle$.

Let $x=(0,0,1) \in H$ and suppose $x=[y, \alpha], y \in H$ as above. Then $y x=y^{\alpha}$, so $(u, v, 1+w)=\left(u \omega, v \omega, w \omega^{2}\right)$. Therefore, $u=v=0$ and $1+w=w \omega^{2}$. Hence $1+w(\omega+1)=w(\omega+1) \omega$. By the above, no such element $w(\omega+1)$ exists. Thus, $x \neq[y, \alpha]$ for any $y$ in $H$, so $x \neq[g, \alpha]$ for any $g$ in $G$. But $x=[(0,1,0),(1,0,0)] \in H^{\prime} \leq G^{\prime}$. Therefore, $\alpha$ is not an anticentral element of $G$. The construction is complete. 
Part c). Let $p$ be an odd prime, $\omega$ a primitive $p$ th root of 1 in the complex numbers $\mathbf{C}$ and $A=\mathbf{Z}[\omega]=\oplus_{0 \leq i<p-1} \mathbf{Z} \omega^{i} \leq \mathbf{C}$. Let $h \in$ Aut $A$ denote multiplication by $\omega$ and let $H=<h>A$ denote the split extension of $A$ by $<h>$.

Define the map $\alpha$ of $H$ into itself by $a \alpha=-a$ for any $a$ in $A$ and

$$
\left(h^{i} a\right) \alpha=h^{i}\left(1+\omega+\cdots+\omega^{i-1}-a\right) \text { for } 0<i<p \text { and } a \in A .
$$

Elementary calculations show that $\alpha$ is a fpf automorphism of $H$ of order 2 .

Recall $G$ denotes the split extension of $H$ by $\langle\alpha>$. Let $\gamma$ denote the map of $G$ into itself given by $g \gamma=[g, \alpha]$. Now $H^{\prime}=\{[a, h]: a \in A\}=(\omega-1) A=A$. Also $h \gamma=1_{A}$ (the multiplicative identity of $A$, not the identity 1.0 of $H$ ), so $G / A$ is abelian. Thus $G^{\prime} \leq A=H^{\prime} \leq G^{\prime}$ and $G^{\prime}=A$. Clearly, $A \gamma=\{-2 a: a \in A\}=2 A$ and $G \gamma=H \gamma$. If $0<i<p$, then

$$
\left(h^{i} a\right) \gamma=(-a) h^{-i} \cdot h^{i}\left(1+\omega+\cdots+\omega^{i-1}-a\right)=1+\omega+\cdots+\omega^{i-1}-2 a .
$$

Hence, $H \gamma \cdot 2 A / 2 A=\left\{2 A, 1+\omega+\cdots+\omega^{i-1}+2 A: 0<i<p\right\}$. Therefore, the cardinality of $H \gamma \cdot 2 A / 2 A$ is $p$. However $\left(G^{\prime}: 2 A\right)=(A: 2 A)=2^{p-1}$. Since $p$ is odd, so $2^{p-1}>p$. Consequently, $G^{\prime} \neq G \gamma$ and hence $\alpha$ is not anticentral in $G$.

If we take $p=2$ in the above construction, the group $G$ and map $\alpha$ do exist with $A$ infinite cyclic, but they do not have the required properties. However, there does exist a suitable example with $(H: A)=2$, but with $A$ of rank 3 , as we now show.

Let $A=<b, c, d>$ be a free abelian group of rank 3. Define $h \in \operatorname{Aut} A$ of order 2 by $b^{h}=c, c^{h}=b \& d^{h}=d^{-1}$ and set $H=<h>A$ (the split extension). Then define $\alpha: H \rightarrow H$ by $a \alpha=a^{-1}$ and $(h a) \alpha=h d a^{-1}$ for all $a$ in $A$. Again simple calculations show that $\alpha$ is a fpf automorphism of $H$ of order 2 .

Let $G=<\alpha>H$ denote the split extension of $A$ by $<\alpha>$. Then $[a, \alpha]=a^{-2}$ and $[h a, \alpha]=d a^{-2}$ for all $a$ in $A$. Therefore

$$
G \gamma=H \gamma=A^{2} \cup d A^{2}=<d>A^{2} .
$$

However, $[b, h]=b^{-1} c \notin<d>A^{2}$. Therefore, $G \gamma \neq G^{\prime}$ and hence $\alpha$ is not anticentral in $G$.

Part d). Let $H=<x, Y>$, where $Y$ is the free abelian group on the $y_{i}$ for $i \in \mathbf{Z}$, the integers, and $x$ acts on $Y$ via $y_{i}^{x}=y_{i+1}$ for each $i$ (so $H$ is the wreath product of two infinite cyclic groups). Define automorphisms $\theta$ and $\phi$ of $H$ by $x \theta=x y_{0}, y \theta=y, x \phi=x$ and $y \phi=y^{-1}$, this for all $y$ in $Y$. Then set $\alpha=\theta \phi \in$ Aut $H$. It is easy to check that $\alpha$ has order 2.

Obviously, $y \alpha=y^{-1}$, so $\alpha$ is fpf on $Y$. Let $r$ be a positive integer. Then

$$
\left(x^{r}\right) \alpha=\left(x y_{0}\right)^{r}=x^{r}\left(y_{0} y_{1} \ldots y_{r-1}\right) \text { and }\left(x^{-r}\right) \alpha=\left(y_{0}^{-1} x^{-1}\right)^{r}=x^{-r}\left(y_{-r} \ldots y_{-2} y_{-1}\right) .
$$

If $y \in Y$ with $\left(x^{r} y\right) \alpha=x^{r} y$, then $x^{r} y_{0} y_{1} \ldots y_{r-1} y^{-1}=x^{r} y$, so $y_{0} y_{1} \ldots y_{r-1} \in Y^{2}$, which is false. If $\left(x^{-r} y\right) \alpha=x^{-r} y$, then we obtain $y_{-r} \ldots y_{-2} y_{-1} \in Y^{2}$, which again is false. Consequently, $\alpha$ is a fpf automorphism of $H$.

Set $G=<\alpha>H$. Now $G^{\prime}$ contains $\left[y_{1}, x\right]=y_{1}^{-1} y_{2} \in y_{1} y_{2} Y^{2}$. Clearly $[y, \alpha]=y^{-2} \in$ $Y^{2}$. Also

$$
\begin{aligned}
& {\left[x^{r} y, \alpha\right]=y^{-1} x^{-r} \cdot x^{r} y_{0} y_{1} \cdots y_{r-1} y^{-1} \in y_{0} y_{1} \cdots y_{r-1} Y^{2} \text { and }} \\
& {\left[x^{-r} y, \alpha\right]=y^{-1} x^{r} \cdot x^{-r} y_{-r} \cdots y_{-2} y_{-1} y^{-1} \in y_{-r} \cdots y_{-2} y_{-1} Y^{2}}
\end{aligned}
$$


Therefore, $\left[y_{1}, x\right] \neq[g, \alpha]$ for all $g \in G, G^{\prime} \neq G \gamma$ in our notation above and hence $\alpha$ is not an anticentral element of $G$. The construction is complete.

Part e). Since $H$ is abelian, $G^{\prime}=[H, \alpha]=H \gamma=G \gamma$, where as above $\gamma$ maps $g$ to $[g, \alpha]$. Therefore $\alpha$ is anticentral in $G$.

Remark 1 Let $H$ be a polycyclic group with a fpf automorphism $\alpha$ of order 2 . Then $A=$ Fitt $H$ is abelian and inverted by $\alpha$ (Part a) of Theorem $1,(H: A)$ is finite ([8, Theorem 1]) and $A=C_{H}(A)([6,2.17])$. If we choose $p=3$ in the construction of the proof of Part c) of Theorem 1, our counter example has $A$ free abelian of rank 2 and $(H: A)=3$. We also have the example with $A$ free abelian of rank 3 and with $(H: A)=2$. These are the smallest possible examples (with $\alpha$ not anticentral in $G$ of course), as the following implies.

Lemma 6 With the above notation, if either A has torsion-free rank (= Hirsch number) at most 1 , or $A$ has torsion-free rank 2 and $(H: A)=2$, then $\alpha$ is anticentral in $G=<\alpha>$ $H$.

Proof Here $A=B \times T$, where $B$ is free abelian of rank at most 2 and $T$ is finite abelian. If $H$ is finite or abelian the claim clearly holds (Lemma 1 and Part e) of Theorem 1), so assume otherwise.

Suppose first that $A$ is free abelian of rank 2 and $(H: A)=2$. Now $H=<h>A$, where $h^{2} \in A$ and $h \alpha=h b$ for some $b$ in $A$. If $a \in A$, then $[a, \alpha]=a^{-2}$ and $[h a, \alpha]=$ $b a^{-2}$. Thus $G \gamma=H \gamma=A^{2} \cup b A^{2}=<b>A^{2}$. If $b=a^{2}$ for some $a$ in $A$, then (ha) $\alpha=h b a^{-1}=h a$, contradicting $\alpha$ fpf. Hence $b \notin A^{2}$.

Now $h^{-2}=\left(h^{2}\right) \alpha=(h \alpha)^{2}=h b h b=h^{2} b^{h} b$. Thus $b^{h} b=h^{-4} \in A^{2}$, so $b^{h} A^{2}=b A^{2}$ and $G \gamma / A^{2}$ lies in the centre $\zeta_{1}\left(H / A^{2}\right)$ of $H / A^{2}$. Clearly $\left|H / A^{2}\right|=8$. If $H / A^{2}$ is abelian, then $H^{\prime} \leq A^{2} \leq G \gamma$. If not, then $H^{\prime} A^{2} / A^{2}=\zeta_{1}\left(H / A^{2}\right)$, which has order 2 . But $b \notin A^{2}$; hence $H^{\prime} A^{2}=G \gamma / A^{2}$. Consequently in either case $H^{\prime} \leq G \gamma$, so $G^{\prime}=H^{\prime} G \gamma=G \gamma$ and $\alpha$ is anticentral in $G$.

Now assume that $A$ is infinite cyclic. Since $A=C_{H}(A)$ and $H$ is not abelian, we have $(H: A)=2$. We can now apply the arguments of the previous case. Alternatively apply the previous case directly to $K=H \times\langle k>$, where $k$ has infinite order and the action of $\alpha$ is extended to $K$ by setting $k \alpha=k^{-1}$. The desired conclusion follows from this and the obvious isomorphism $G \cong<\alpha>K /<k>$.

Finally, consider the general case where $A=B \times T, B$ and $T$ being as above. Now $T$ has odd order since $\alpha$ is fpf, so $\alpha$ is fpf on $H / T$ by [9, Lemma $13 \mathrm{c}$ )]. Thus $G \gamma T / T=$ $G^{\prime} T / T \leq A / T$ by the previous two cases. If $t \in T$, then $t$ has odd order and hence $t=s^{-2}$ for some $s \in T$. Then $[s, \alpha]=t$ and $(g s) \gamma=g \gamma \cdot t$ for any $g$ in $G$. Consequently, $G^{\prime} T=G^{\prime}$ and $G \gamma \cdot T=G \gamma$. Therefore, $\alpha$ is anticentral in $G$.

Open Access This article is distributed under the terms of the Creative Commons Attribution 4.0 International License (http://creativecommons.org/licenses/by/4.0/), which permits unrestricted use, distribution, and reproduction in any medium, provided you give appropriate credit to the original author(s) and the source, provide a link to the Creative Commons license, and indicate if changes were made.

\section{References}

1. Ersoy, K.: Infinite groups with an anticentral element. Comm. Algebra 40, 4627-4638 (2012)

2. Ersoy, K.: Finite groups with a splitting automorphism of odd order. Arch. Math. 106, 401-407 (2016) 
3. Gorenstein, D.: Finite Groups. Harper \& Row, New York (1968)

4. Ladisch, F.: Groups with anticentral elements. Comm. Algebra 36, 2883-2894 (2008)

5. Lennox, J.C., Robinson, D.J.S.: The Theory of Infinite Soluble Groups. Clarendon Press, Oxford (2004)

6. Robinson, D.J.S.: Finiteness Conditions and Generalized Soluble Groups (2 volumes). Springer, Berlin (1972)

7. Wehrfritz, B.A.F.: Infinite Linear Groups. Springer, Berlin (1973)

8. Wehrfritz, B.A.F.: Almost fixed-point-free automorphisms of order 2. Rend. Circ. Mat. Palermo 60, 365-370 (2011)

9. Wehrfritz, B.A.F.: Almost fixed-point-free automorphisms of prime order. Cent. Eur. J. Math. 9, 616-626 (2011)

10. Wehrfritz, B.A.F.: On the fixed-point set of an automorphism of a group. Publ. Mat. 57, 139-153 (2013)

11. Wehrfritz, B.A.F.: Automorphisms of finite order of nilpotent groups. Ricerche Mat. 63, 261-272 (2014) 